\title{
Radio Frequency Interference Measurements for a Radio Astronomy Observatory Site in Indonesia
}

\author{
Peberlin Parulian Sitompul *, Timbul Manik (D, Mario Batubara and Bambang Suhandi \\ Space Science Center, National Institute of Aeronautics and Space of Indonesia, 40173 Bandung, Indonesia; \\ timbul.manik@lapan.go.id (T.M.); mario.batubara@lapan.go.id (M.B.); bambang.suhandi@lapan.go.id (B.S.) \\ * Correspondence: peberlin.sitompul@lapan.go.id
}

Citation: Sitompul, P.P.; Manik, T.; Batubara, M.; Suhandi, B. Radio Frequency Interference Measurements for a Radio Astronomy Observatory Site in Indonesia. Aerospace 2021, 8, 51. https://doi.org/10.3390/ aerospace 8020051

Received: 8 January 2021

Accepted: 5 February 2021

Published: 17 February 2021

Publisher's Note: MDPI stays neutral with regard to jurisdictional claims in published maps and institutional affiliations.

Copyright: (c) 2021 by the authors. Licensee MDPI, Basel, Switzerland. This article is an open access article distributed under the terms and conditions of the Creative Commons Attribution (CC BY) license (https:// creativecommons.org/licenses/by/ $4.0 /)$.

\begin{abstract}
We report on the measurements of radio frequency interference (RFI) at Mount Timau, Kupang, Indonesia, which is intended to host a future radio astronomy observatory. These measurements were taken twice in October 2020 and December 2020 to obtain the RFI environment, at frequencies between 70 and $7000 \mathrm{MHz}$. Due to the limitations of the measurement data, the results presented in this paper are based on peak detection rather than statistical analysis. Based on the measurement results, the frequency interval between $70-88 \mathrm{MHz}$ and $120-150 \mathrm{MHz}$ is relatively quiet, and the frequency range of $150-300 \mathrm{MHz}$ is relatively clear. The frequency interval of 300 to $800 \mathrm{MHz}$ is relatively quiet, except at the frequency of $600 \mathrm{MHz}$. The frequency range of $800-1400 \mathrm{MHz}$ is also relatively quiet. The predominant terrestrial services in this band are at $840 \mathrm{MHz}$, with an amplitude around $32 \mathrm{~dB}$, and $916 \mathrm{MHz}$, with an amplitude around $12 \mathrm{~dB}$, and the global system for mobile (GSM) signals around $954 \mathrm{MHz}$ have an amplitude around $20 \mathrm{~dB}$ above the noise floor. The frequency range of $1400-7000 \mathrm{MHz}$ is also relatively quiet. In this band frequency, we can see RFI at 2145 and $2407 \mathrm{MHz}$, emitted by local Wi-Fi, and at $2683 \mathrm{MHz}$, with amplitudes of 18, 40 and $15 \mathrm{~dB}$, respectively, from the noise level. We conclude that, for this period, the frequency band allocated for astronomy can possibly be used for radio telescope development.
\end{abstract}

Keywords: radio frequency interference; radio astronomy; radio wave

\section{Introduction}

Presently, man-made radio frequency interference (RFI) is continuously increasing in telecommunication systems, radar systems, radio navigation services etc. [1-4]. These RFIs will affect the radio astronomy data obtained for astronomic research. For mitigation, RFI detection is underway and mitigation methods are being planned, meaning that more reliable measurements can be obtained.

Site evaluation for an astronomical observatory in Indonesia is being performed as part of a feasibility study for a new radio telescope at a multi-wavelength radio frequency observatory. The East Nusa Tenggara, located in southeastern Indonesia, holds the most promising candidate locations for such an astronomical site, based on its clear sky fraction [5]. The radio spectrum is becoming increasingly occupied, mainly at frequencies below $2 \mathrm{GHz}$, most commonly by wireless communications [1]. As such, it is important to conduct RFI measurements to ascertain the suitability of certain locations for a radio telescope system. The measured data can be used as references for RFI measurements in the future. For the present RFI measurement, we selected the frequency range used for radio astronomy: in $70 \mathrm{MHz}-7 \mathrm{GHz}$. Knowing the RFI conditions in several specified frequency bands is very important, since this will provide information on its measured levels and the procedure for its mitigation. It is also very useful for interpreting the corresponding data.

The RFI in our measurements could possibly be generated by television and radio broadcasts, the global system for mobile (GSM) communication, amateur radio communication, automotive emissions, radio navigation, aeronautical radar, and so on [1-3]. In 
Indonesia, the regulations of frequency allocation are managed by the Ministry of Communication and Informatics [6,7]. Our measurements could be useful as a reference to check if the frequency band allocations of the regulation office are really being followed. The policy of radio frequency allocation is regularly reviewed by this ministry, and basically states compulsory compliance to the International Telecommunication Union (ITU) recommendation. This paper is organized as follows. An introduction is presented in Section 1. A detailed description of the locations, instruments and methods involved in obtaining the measurements is presented in Section 2. The measurement results and their analysis are presented in Section 3, and the conclusions are given in Section 4.

\section{The RFI Measurement}

\subsection{Location of RFI Measurement}

The locations for radio telescopes are selected to be as far away as possible from populated areas. Considering the meteorological conditions described in Ref. [5], the location selected for our measurement is the Mount Timau area, which is a mountainous site. The site where the construction of an optical telescope is now underway is shown in Figure 1.

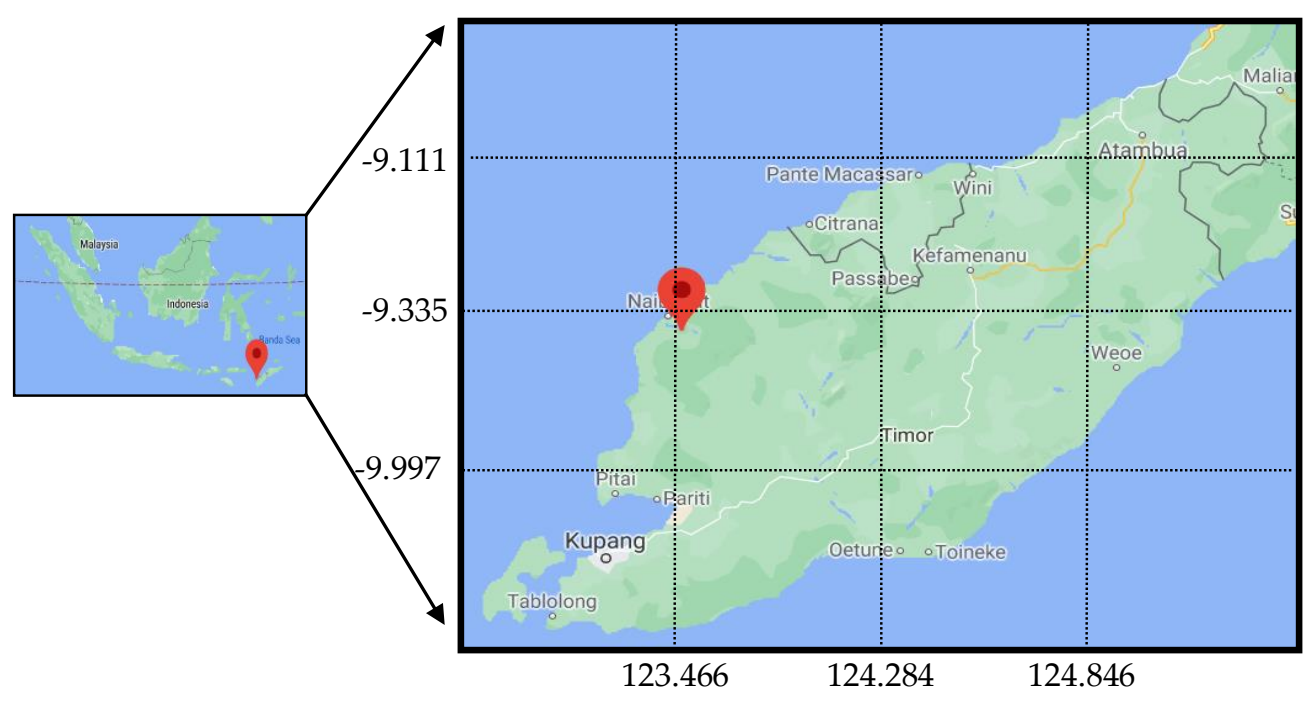

Figure 1. Location of national observatory [8].

Figure 1. shows the location for RFI measurements-Timau Mount, at longitude (E) $123^{\circ} 56^{\prime} 24.4^{\prime \prime}$, latitude (S) $9^{\circ} 35^{\prime} 4.9^{\prime \prime}$, and altitude $1532 \mathrm{~m}$.

\subsection{Instruments for RFI Measurement}

Two types of spectrum analyzers were used in this measurement: (1) Spectrum MasterMS2721B, Anritsu Company, California, USA, working in the frequency interval of $10-7100 \mathrm{MHz}$, for the measurements obtained in October 2020. (2) Spectrum Analyzer DSA 815, Rigol Technologies Inc., Beijing, China, working in the frequency interval of 0.9-1500 MHz, with a displayed average noise level (DANL) of $-136 \mathrm{dBm}$ and a minimum resolution bandwidth (RBW) of $100 \mathrm{~Hz}$, for the measurements obtained in December 2020. The antenna used in this work were log periodic antennas with a working frequency of 40-1800 MHz, and a dipole low-gain antenna. For the second measurement, we used a preamplifier around $13 \mathrm{~dB}$ that was installed on the antenna boom, connected at around $3 \mathrm{~m}$ from the antenna connector output.

Commonly, RFI signals are polarized [8], so measuring the polarization of the RFI is important. In this measurement, we also activated the pre-amplifier prepared inside the spectrum analyzer. Photos of the measurement are shown in Figure 2, with two types of spectrum analyzers-a log periodic and a dipole antenna. 


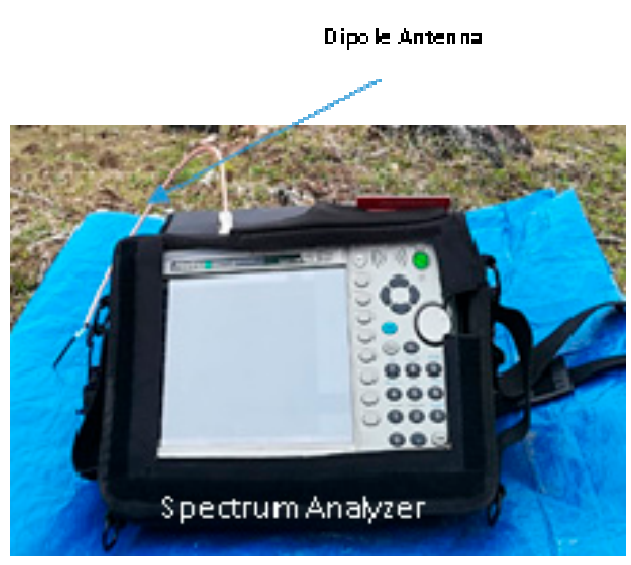

(a)

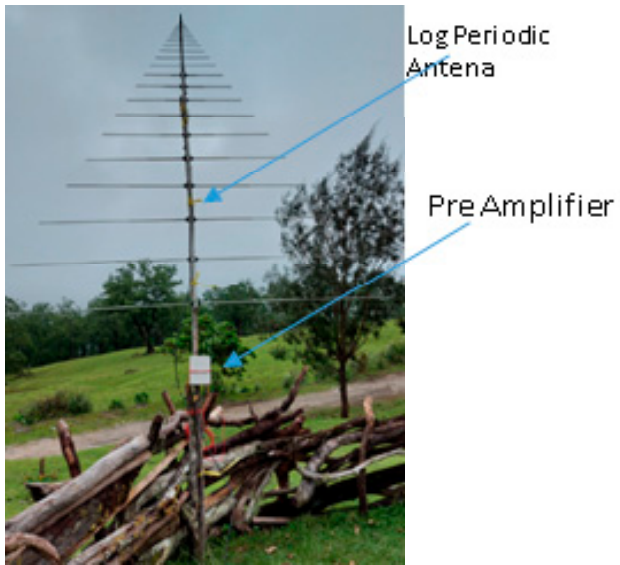

(b)

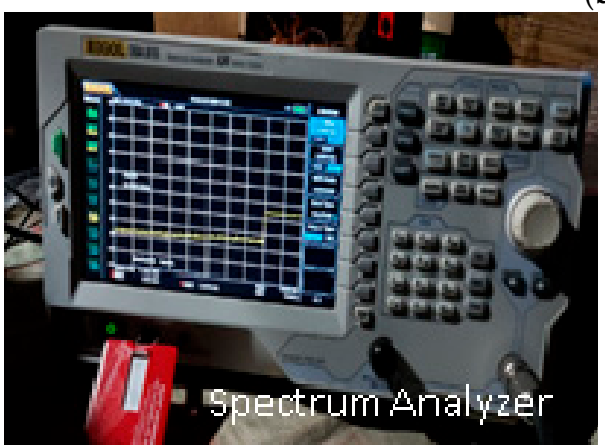

(c)

Figure 2. Photos of measurement: (a) Anritsu MS2721B; (b) log periodic antenna and pre amplifier; (c) Rigol DSA 815 .

In the measurement process, we applied the Square Kilometre Array (SKA) protocol, as described in Ref. [9], as a guidance, although not always in the same way. The SKA protocol covers a frequency range from $70 \mathrm{MHz}$ to $22 \mathrm{GHz}$. The sensitivity of our measurement system and the gain of the antennae were lower than that specified by the protocol, and our measurement cycle was also shorter.

The SKA protocol divides the radio interference into two broad classes [9]: strong radio interference and weak radio interference. Part of the specification for weak radio inference, and the frequency interval commonly used for radio astronomy, are shown in Table 1.

Table 1. Measurement cycle specified by the SKA protocol, for Mode 1 adapted from $[9,10]$. RBW: minimum resolution bandwidth in $\mathrm{KHz}$ units.

\begin{tabular}{llll}
\hline $\begin{array}{l}\text { Frequency Band } \\
\mathbf{( M H z )}\end{array}$ & $\begin{array}{l}\text { RBW } \\
\mathbf{( K H z )}\end{array}$ & $\begin{array}{l}\text { Frequency } \\
\mathbf{( M H z )}\end{array}$ & For Observation \\
\hline $10-150$ & 3 & 38 & $\begin{array}{l}\text { Observation of the Sun, Jupiter, } \\
\text { interstellar medium and steep } \\
\text { spectrum sources }\end{array}$ \\
\hline $150-300$ & 3 & $150-153$ & $\begin{array}{l}\text { Sun, interstellar medium, } \\
\text { pulsar and continuum } \\
\text { observation }\end{array}$ \\
\hline $300-800$ & 30 & $322-328.6,406.1-410$, & $\begin{array}{l}\text { Sun, interstellar medium, } \\
\text { deuterium lines, steep } \\
\text { spectrum sources and pulsar }\end{array}$ \\
\hline
\end{tabular}


Table 1. Cont.

\begin{tabular}{|c|c|c|c|}
\hline $\begin{array}{l}\text { Frequency Band } \\
\text { (MHz) }\end{array}$ & $\begin{array}{l}\text { RBW } \\
\text { (KHz) }\end{array}$ & $\begin{array}{l}\text { Frequency } \\
\text { (MHz) }\end{array}$ & For Observation \\
\hline $800-960$ & 30 & & $\begin{array}{l}\text { For observing redshifted line } \\
\text { from extragalactic sources }\end{array}$ \\
\hline $960-1400$ & 1000 & $\begin{array}{ll}\text { 1. } & 1420.4 \\
\text { 2. } & 1300-1400\end{array}$ & $\begin{array}{ll}\text { 1. } & \text { Rest frequency of neutral } \\
\text { hydrogen }(\mathrm{HI}) \text { at } \\
\text { 1420.4 MHz } \\
\text { 2. } \\
\text { Extragalactic HI and } \\
\text { recombination lines } \\
\text { observations }\end{array}$ \\
\hline $1400-3000$ & 30 & 1420.4 & $\begin{array}{l}\text { Neutral hydrogen }(\mathrm{HI}) \text { at } \\
1420.4 \mathrm{MHz}\end{array}$ \\
\hline $3000-22,000$ & 1000 & $\begin{array}{ll}\text { 1. } & 3252.9-3352.5 \\
\text { 2. } & 4813.6-4834.5 \\
\text { 3. } & 6661.8 \\
\text { 4. } & 4800-5000\end{array}$ & $\begin{array}{ll}\text { 1. } & \text { Methyladyne }(\mathrm{CH}) \\
\text { 2. } & \text { Formaldehyde }\left(\mathrm{H}_{2} \mathrm{CO}\right) \\
\text { 3. } & \text { Methanol }\left(\mathrm{CH}_{3} \mathrm{OH}\right) \\
\text { 4. } & \text { Continuum observation }\end{array}$ \\
\hline
\end{tabular}

\section{Results and Discussion}

We took two measurements at Mount Timau, in October 2020 and December 2020, in the night and the day time. The location was very close to the summit of Mount Timau. For these measurements, we use a handheld spectrum analyzer with a dipole low-gain antenna and a log periodic antenna. At the altitude of $1532 \mathrm{~m}$ above sea level, the line of sight to Kupang City, a large city, is around $70 \mathrm{~km}$, and that to Dili, Timor-Leste is around $200 \mathrm{~km}$. These are possible strong emitters of the RFI that is picked up at the site. Here, measurements were made to cover the radio frequency of 70-7000 MHz.

The measurement results are plotted in Figures 3-12 and are expressed as power spectral intensity in $\mathrm{dBm}$ versus frequency in $\mathrm{MHz}$. The analysis is also discussed below.

\subsection{Band 1: $70-150 \mathrm{MHz}$}

The 70-150 MHz range is the lowest frequency range assigned within the SKA protocol and is referred to as Band 1. We can see from Figure 3, that terrestrial services contribute to most of the RFI. A significant share of the RFI in this band originates from radio broadcasts for frequency modulation (FM) radio, in the $88-118 \mathrm{MHz}$ range, with amplitude varying from 15 to $40 \mathrm{~dB}$ above the noise floor. The frequency between $70-88 \mathrm{MHz}$ and $120-150 \mathrm{MHz}$ is relatively quiet.

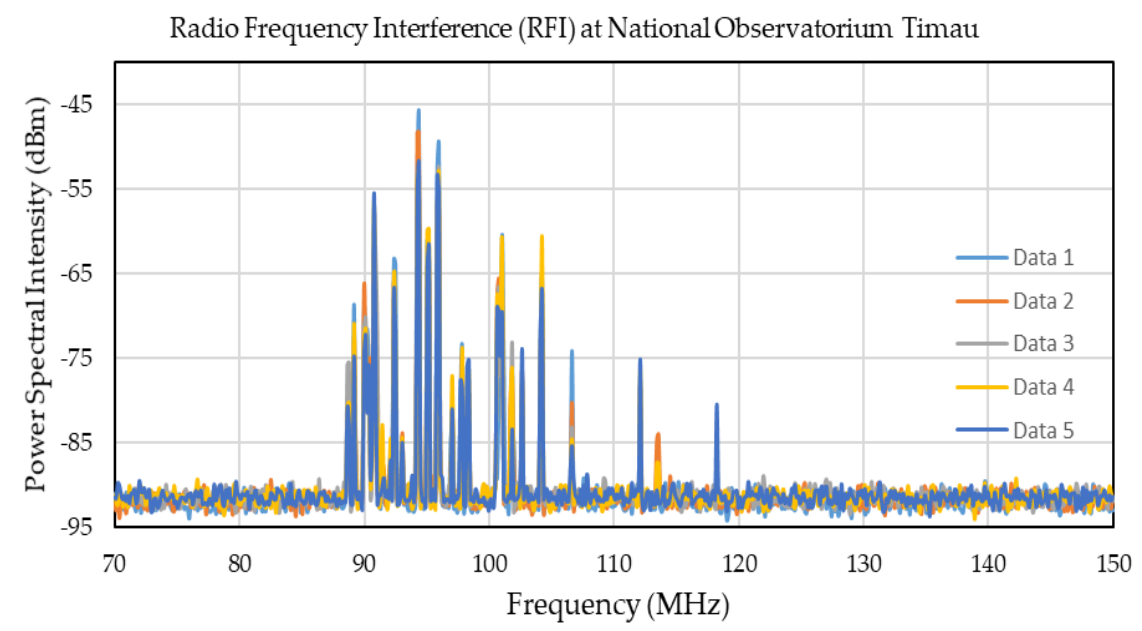

Figure 3. Results of a radio frequency survey at low frequencies between 70 and $150 \mathrm{MHz}$. 


\subsection{Band 2: $150-300 \mathrm{MHz}$}

At Mount Timau, Band 2, with the frequency range of $150-300 \mathrm{MHz}$, is relatively clear, and there is no strong RFI in this band except at the frequency of $287 \mathrm{MHz}$, with an amplitude of $6 \mathrm{~dB}$ above the noise floor. The vertical axis in Figure 4 depicts from -100 to $-80 \mathrm{dBm}$, in order to enhance the very weak signal at $287 \mathrm{MHz}$. However, the RFI signals at $260 \mathrm{MHz}$, from military geostationary satellites, are undetected, probably due to the low sensitivity of our measurement equipment.

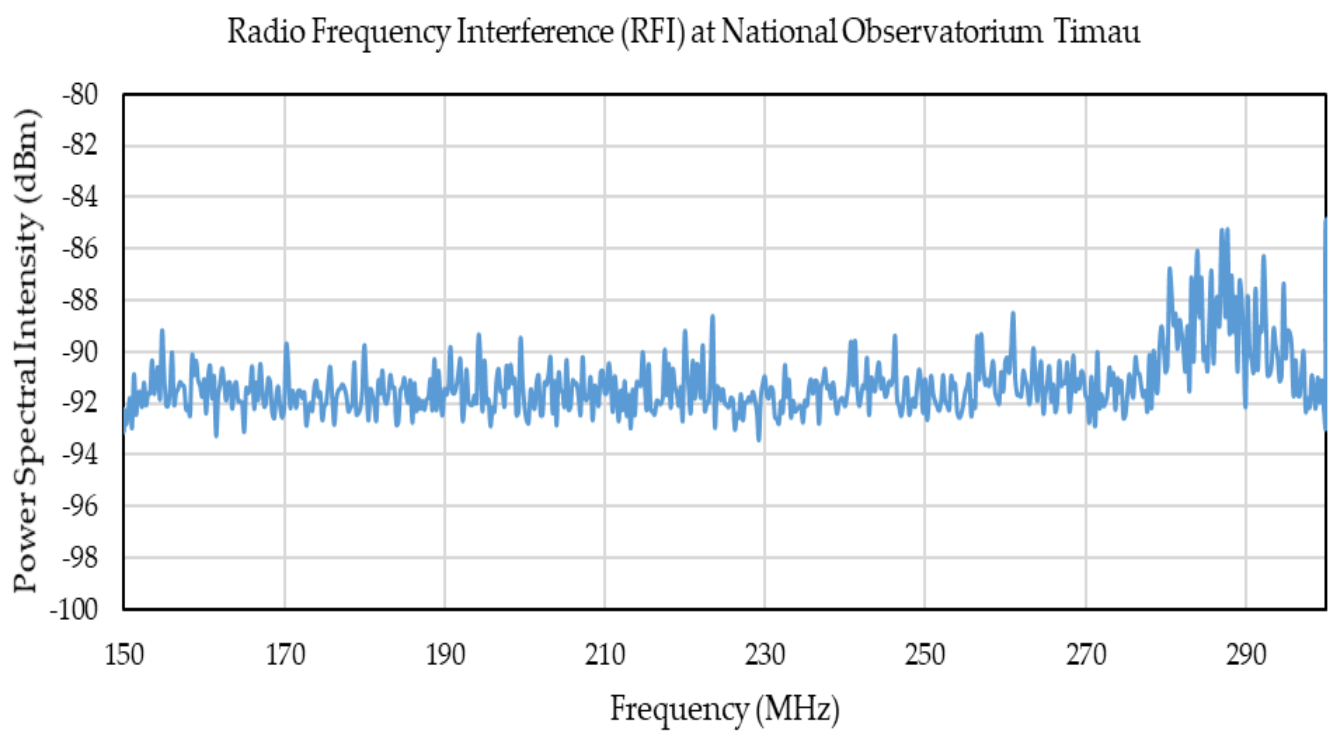

Figure 4. Results of radio frequency survey at low frequencies between 150 and $300 \mathrm{MHz}$.

\subsection{Band 3: 300-800 MHz}

The frequency interval of 300 to $800 \mathrm{MHz}$ is very important for radio astronomy, as regards observing the interstellar medium, the Sun, deuterium lines, steep spectrum sources, and pulsars in the frequencies $322-328.6 \mathrm{MHz}, 406.1-410 \mathrm{MHz}$, and 608-614 MHz [10,11]. However, these interval frequencies are also allocated for other science services, such as for communication, and biomass and soil measurements by National Oceanic and Atmospheric Administration (NOAA) [11]. We noticed that this band is busy in the frequency range of 336-554 MHz, at the average power spectral intensity of $8 \mathrm{dBm}$, and with the maximum power spectral intensity of $15 \mathrm{dBm}$ at $536 \mathrm{MHz}$. The RFI here is mostly caused by television broadcasts, wireless communication and land mobile communication [6,7]. In the manual of Indonesian frequency allocation [6,7], this band is also reserved for universal service obligation, public protection and disaster relief, international mobile telecommunication, and high-definition television. In Figure 5, we can see RFI in the range of 336-554 MHz, even though this has relatively low amplitude; however, the frequency range from 600 to $800 \mathrm{MHz}$ still contains many frequency slots that can be used for radio astronomy.

\subsection{Band 4: $800-960 \mathrm{MHz}$}

In Band 4, with the frequency interval of $800-960 \mathrm{MHz}$, there are no special allocation bands for radio astronomy. However, this band is used to observe the redshifted lines emitted by extragalactic sources. In our measurements, the resolution bandwidth (RBW) was set to $30 \mathrm{KHz}$, and the noise floor rose to about $-85 \mathrm{dBm}$. We managed to perform three measurement cycles. The terrestrial service allocations in this band are mainly for mobile communication and fixed wireless access, at $840 \mathrm{MHz}$, with an amplitude around $32 \mathrm{~dB}$ above the noise floor, and $916 \mathrm{MHz}$, with an amplitude around $12 \mathrm{~dB}$ above the noise floor, respectively. The GSM signals around $954 \mathrm{MHz}$ have an amplitude around $20 \mathrm{~dB}$ above the noise floor, as shown in Figure 6. We also observed a radio signal at $871 \mathrm{MHz}$ that suddenly appeared and disappeared. 
Radio Frequency Interference (RFI) at National Observatorium Timau

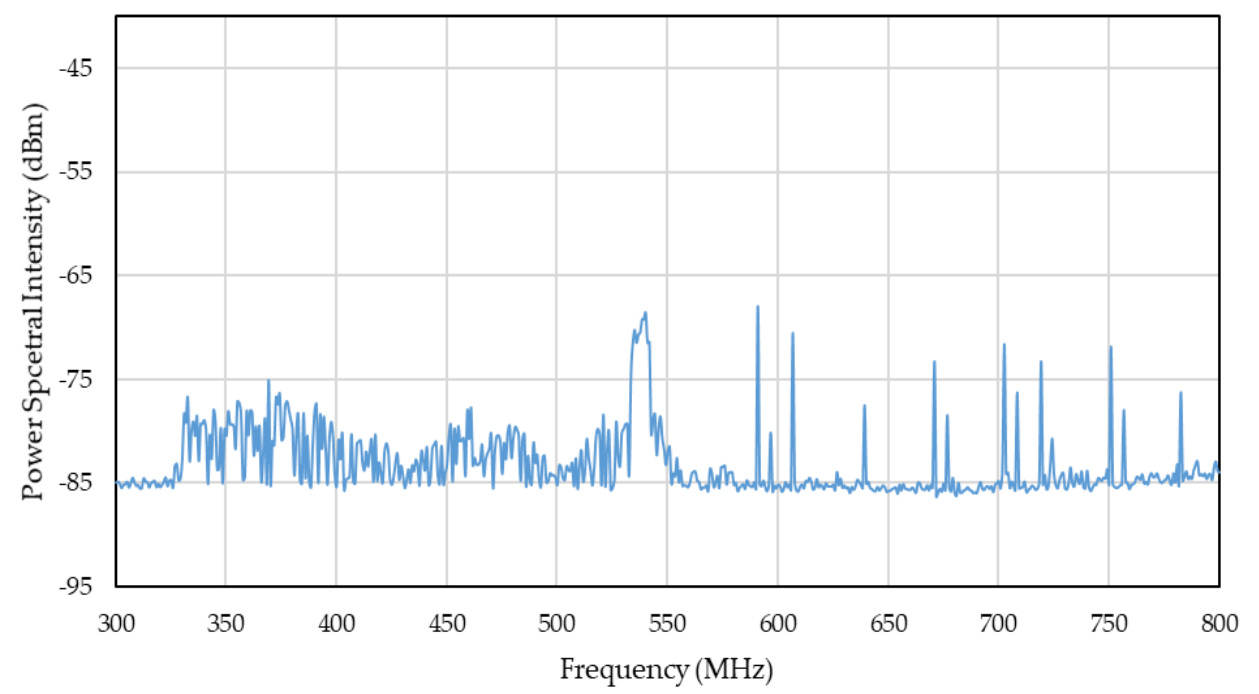

Figure 5. Results of radio frequency survey at low frequencies between 300 and $800 \mathrm{MHz}$.

Radio Frequency Interference (RFI) at National Observatorium Timau

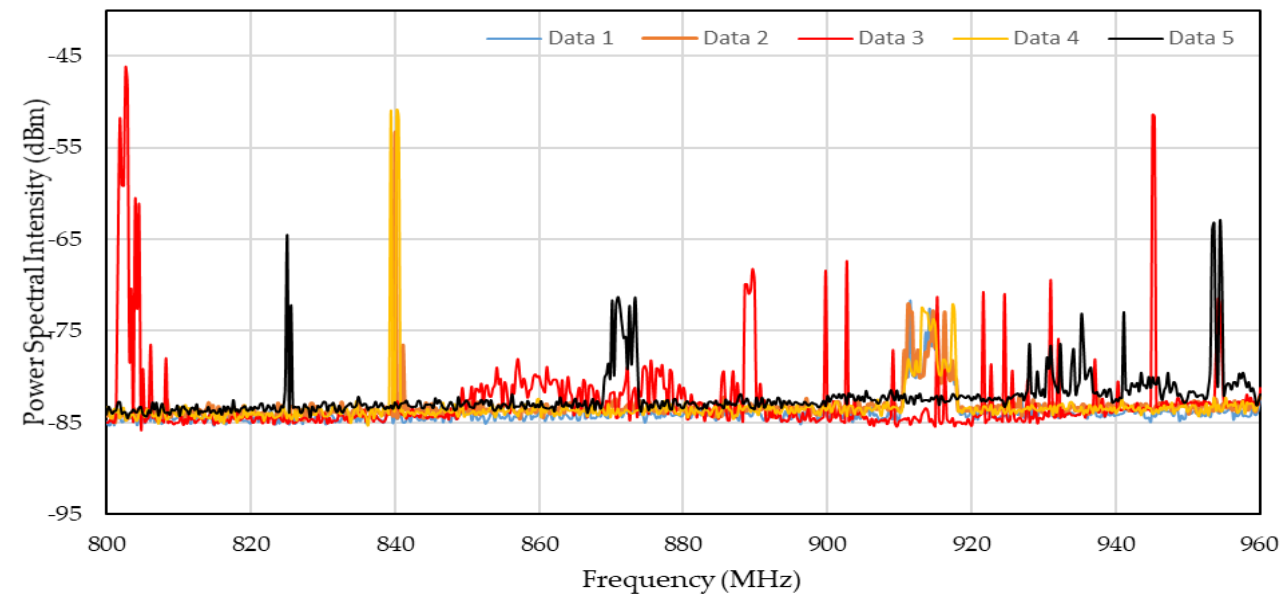

Figure 6. Results of radio frequency survey at low frequencies between 800 and $960 \mathrm{MHz}$.

\subsection{Band 5: 960-1400 MHz}

The rest frequency of neutral hydrogen (HI) is $1420.4 \mathrm{MHz}$. As such, the frequencies in the 960-1400 MHz range close to the HI frequency are very important for radio astronomy. Extragalactic observations must consider the redshifted lines that fit in this frequency band. The frequencies from 1300 to $1400 \mathrm{MHz}$ are allocated for extragalactic $\mathrm{HI}$ and recombination line observations $[10,11]$. From Figure 7 , we can see that this band area is very quiet.

\subsection{Band 6: 1400-2000 MHz}

The frequency range of 1400 to $2000 \mathrm{MHz}$ is also very important for radio astronomy as regards the observation of galactic and local extragalactic neutral hydrogen (HI), recombination lines, radio source spectra, the galactic continuum, and extragalactic $\mathrm{OH}$ masers $[10,11]$. The frequency ranges of 1428-1452 MHz and 1498-1522 MHz are actually allocated for wireless broadband [2-7], and other sub bands for mobile satellite communication. 
Radio Frequency Interference (RFI) at National Observatorium Timau

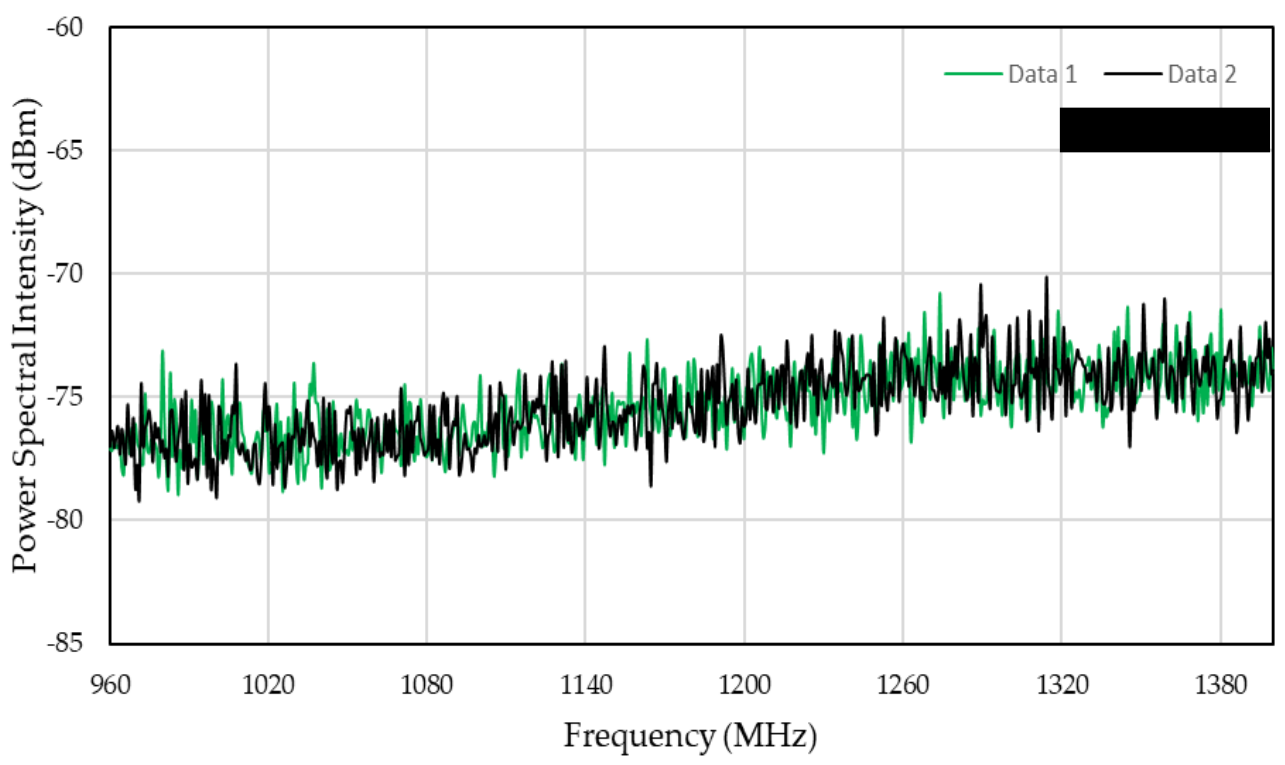

Figure 7. Results of radio frequency survey at low frequencies between 960 and $1400 \mathrm{MHz}$.

We note the presence of strong mobile communication signals at $1650.9 \mathrm{MHz}$, with an amplitude around $22 \mathrm{~dB}$ from the noise floor. In the frequency band of $1836-1876 \mathrm{MHz}$, we also found strong RFI with amplitudes over $25 \mathrm{~dB}$ from the noise floor and bandwidth around $50 \mathrm{MHz}$, as shown on Figure 8, this being emitted by a Very-small-aperture terminal (VSAT) for internet services.

According to the ITU recommendation RA.769-2, Band 7, with the frequency interval of 2000-3000 MHz, is reserved for radio astronomy and other ISM (industrial, scientific and medical) purposes. In this frequency band, we can see RFI at $2145 \mathrm{MHz}$ and $2407 \mathrm{MHz}$, emitted by local $\mathrm{Wi}-\mathrm{Fi}$, and at $2683 \mathrm{MHz}$, with amplitudes of 18,40 and $15 \mathrm{~dB}$ from the noise level, respectively, as shown in Figure 9.

Radio Frequency Interference (RFI) at National Observatorium Timau

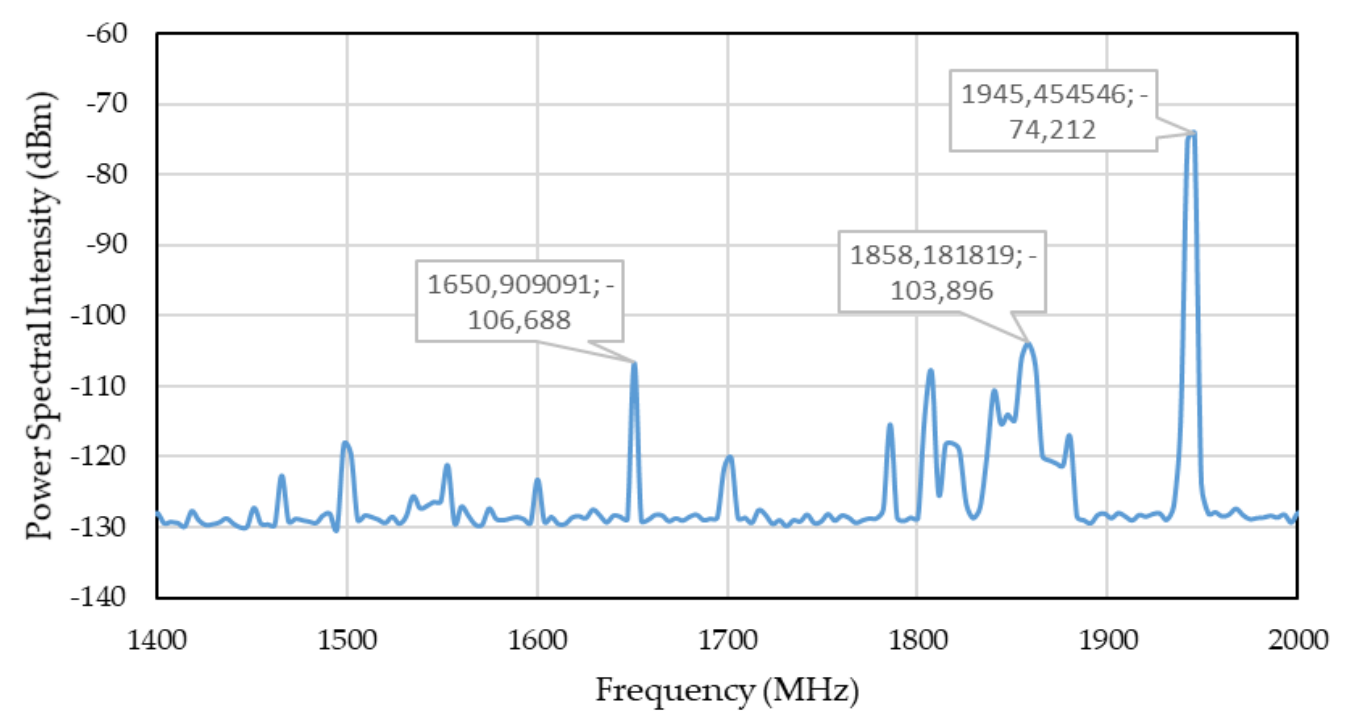

Figure 8. Results of radio frequency survey at low frequencies between 1400 and $2000 \mathrm{MHz}$. 


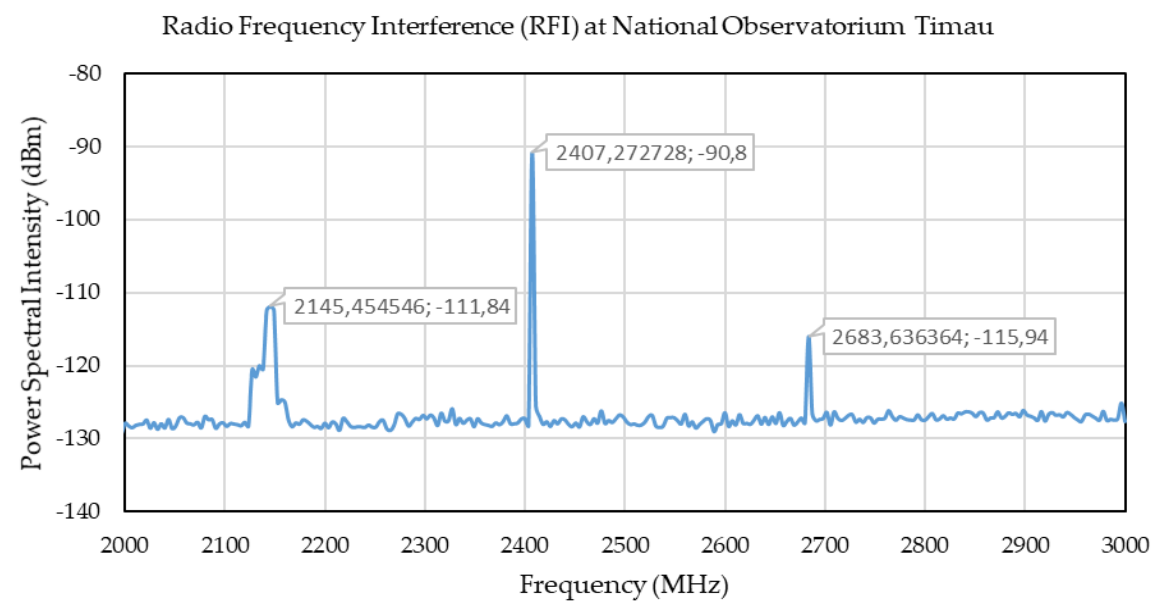

Figure 9. Results of radio frequency survey at low frequencies between 2000 and $3000 \mathrm{MHz}$.

\subsection{Bands 8 and 9: $4000-7000 \mathrm{MHz}$}

Bands 8 and 9, with a frequency range of $3000-8000 \mathrm{MHz}$, are also important for radio astronomy. The measurement of RFI in these bands is displayed in Figure 10a,b. None of the predominant terrestrial RFI sources are observed in this frequency range. It is worth noting that the measurement at these frequencies was undertaken using two antenna types; the results in Figure 10a were derived using a log periodic antenna, and those in Figure 10b using a dipole antenna, as in Figure 2, for the comparison of measurement. These results are less accurate due to the limitation of the antenna bandwidth, based on technical specification.

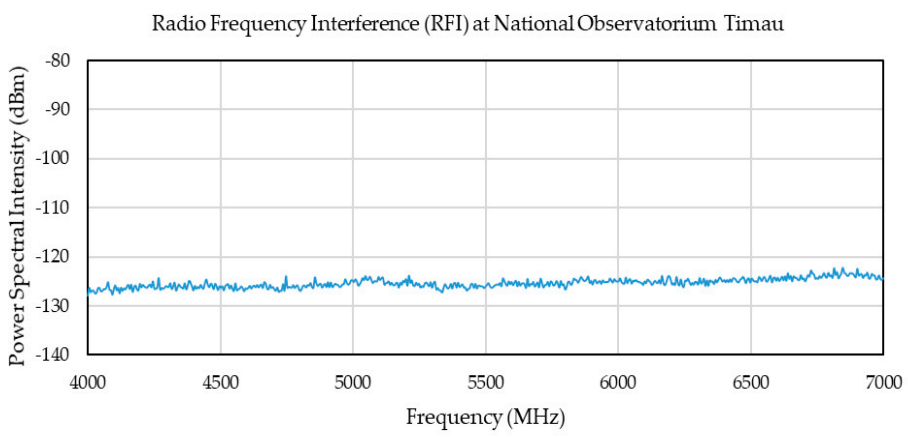

(a)

Radio Frequency Interference (RFI) at NationalObservatorium Timau

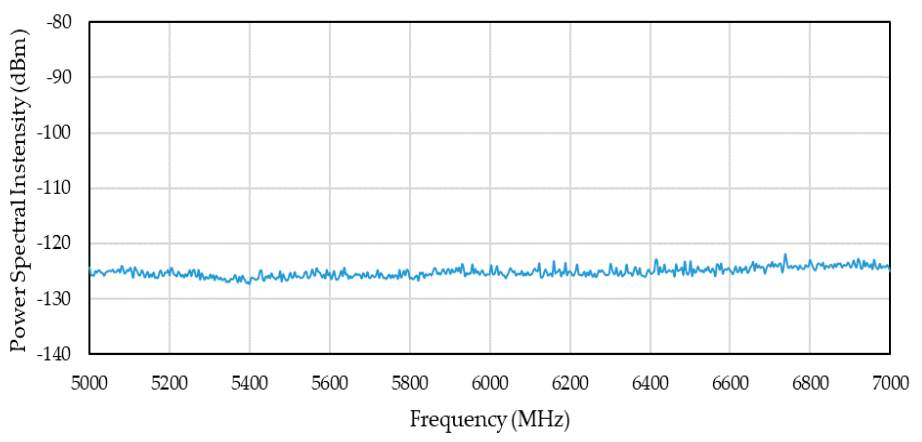

(b)

Figure 10. Results of radio frequency measurement at the following frequencies: (a) between 4000 and $7000 \mathrm{MHz}$, using a log-periodic antenna; (b) between 5000 and $7000 \mathrm{MHz}$, using a dipole antenna. 
Radio Frequency Interference (RFI) at National Observatorium Timau

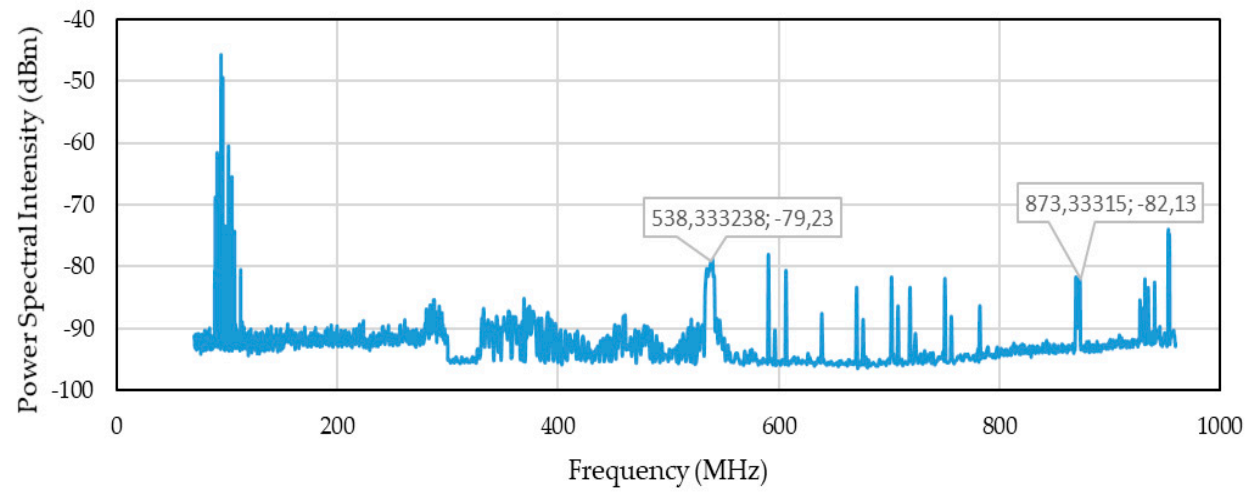

Figure 11. Recent measurements at frequencies 70-1000 MHz.

Radio Frequency Interference (RFI) at National Observatorium Timau

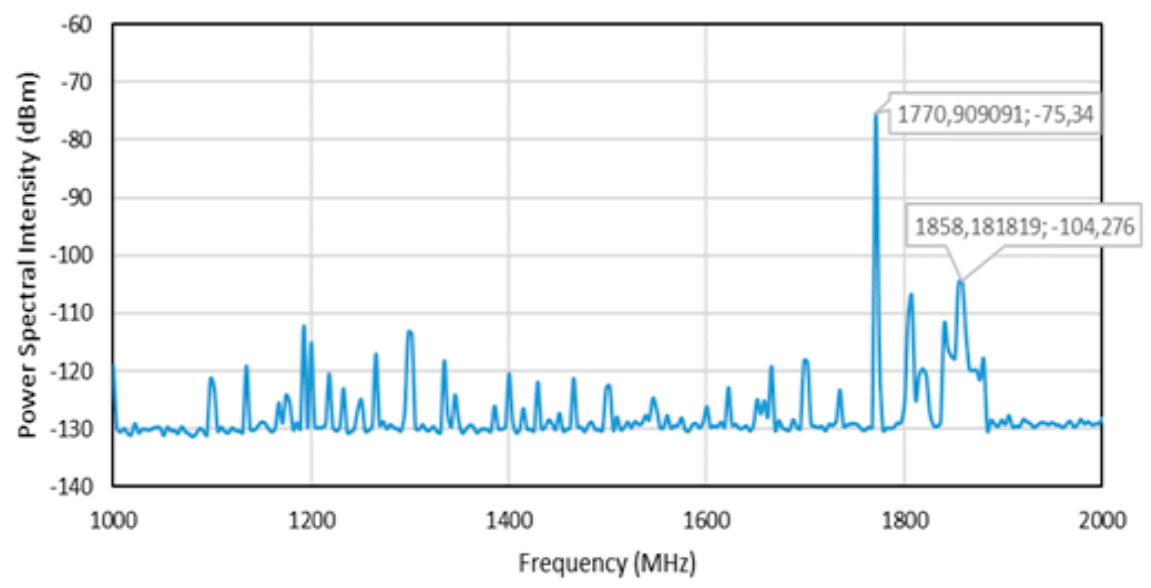

Figure 12. Recent measurements at frequencies 1000-2000 MHz.

In Figures 11 and 12, we compare the RFI results from previous measurements [12] in the frequency ranges of 70-1000 MHz and 1000-2000 MHz. In Figure 12, we can see a great deal of RFI, mainly at $1.8 \mathrm{GHz}$, which is generated by Wi-Fi in the area.

For comparison, the RFI measured in other countries [13] was emitted by radio FM at frequencies of $87-108 \mathrm{MHz}$, by television broadcasting at frequencies of $510-790 \mathrm{MHz}$, by mobile communication at frequencies of $870-960 \mathrm{MHz}$ and $1805-2170 \mathrm{MHz}$, and by Wi-Fi at frequencies of 2306-2481 MHz.

\section{Conclusions}

From this work, we can conclude that the RFI environment at Mount Timau is very quiet, except in the Very High Frequency (VHF) and Ultra High Frequency (UHF) bands. The frequency ranges of 70-88 MHz and 120-150 MHz are relatively quiet. In Band 2, the frequency range of $150-300 \mathrm{MHz}$ is also relatively clear. This band is used for Sun, interstellar medium, pulsar and continuum observations. The frequency interval of 300 to $800 \mathrm{MHz}$ is important for radio astronomy, in terms of the observation of the interstellar medium, the Sun, deuterium lines, steep spectrum sources, and pulsars with the frequencies of 322-328.6 MHz, 406.1-410 MHz, and 608-614 MHz. We can see from Figure 8 that this band is relatively quiet, except at the frequency of $600 \mathrm{MHz}$. The frequency ranges of 960-1400 MHz, close to the HI frequency, is also relatively quiet. The frequency ranges of 1400-7000 MHz, used for neutral hydrogen (HI) (at $1420.4 \mathrm{MHz}$ ), methyladyne $(\mathrm{CH})$, formaldehyde $\left(\mathrm{H}_{2} \mathrm{CO}\right)$, methanol $\left(\mathrm{CH}_{3} \mathrm{OH}\right)$ and continuum observations, is also relatively quiet. 
Author Contributions: Conceptualization, P.P S. and T.M.; methodology, P.P.S.; software, P.P.S.; validation, P.P.S., T.M., M.B. and B.S.; formal analysis, P.P.S. and M.B.; investigation, P.P.S. and M.B.; resources, P.P.S., T.M., M.B. and B.S.; data curation, P.P.S., M.B.; writing—original draft preparation, P.P.S.; writing - review and editing, P.P.S., T.M. and M.B.; visualization, P.P.S.; supervision, T.M. and B.S.; project administration, P.P.S.; funding acquisition, P.P.S. and T.M. All authors have read and agreed to the published version of the manuscript.

Funding: This research was funded by National Institute of Aeronautics and Space of Indonesia (LAPAN), Indonesia.

Acknowledgments: The authors wish to thank the staff at Management Center of National Observatory, LAPAN, Kupang for their kind support of this program, Bambang Siantoro for his kind support in providing the spectrum analyzer and tools for measurement, and Ruddy Kurniawan for help with the measurements. The funding support from LAPAN is also greatly appreciated. The authors acknowledge the anonymous reviewers for the very helpful and useful comments to improve this paper.

Conflicts of Interest: The authors declare no conflict of interest.

\section{References}

1. Chen, D.; Yang, J.; Wu, J.; Tang, H.; Huang, M. Spectrum occupancy analysis based on radio monitoring network. In Proceedings of the 1st IEEE International Conference on Communication, Beijing, China, 15-17 August 2012. Wireless Networking Application.

2. Islam, H.; Koh, C.L.; Oh, S.W.; Qing, X.; Lai, Y.Y.; Wang, C.; Liang, Y.-C.; Toh, B.E.; Chin, F.; Tan, G.L.; et al. Spectrum survey in Singapore: Occupancy measurements and analyses. In Proceedings of the International Conference on Cognitive Radio Oriented Wireless Networks and Communications (CROWNCOM), Singapore, 15-17 May 2008.

3. Kucuk, I.; Uler, I.; Oz, S.; Onay, S.; Ozdemir, A.R.; Gulcsen, M.; Sarikaya, M.; Dagtekin, N.D.; Ozeren, F.F. Site selection for a radio astronomy observatory in Turkey: Atmospherical, meteorological, and radio frequency analyses. Exp. Astron. 2012, 33, 1-26. [CrossRef]

4. Abidin, Z.Z.; Adnan, S.; Ibrahim, Z.A. RFI profiles of prime candidate sites for the first radio astronomical telescope in Malaysia. New Astron. 2010, 15, 307-312. [CrossRef]

5. Hidayat, T.; Mahasena, P.; Dermawan, B.; Hadi, T.W.; Premadi, P.W.; Herdiwijaya, D. Clear sky fraction above Indonesia: An analysis for astronomical site selection. Mon. Not. R. Astron. Soc. 2012, 427, 1903-1917. [CrossRef]

6. Regulation of the Minister of Communication and Informatics. No. 29/PER/M.KOMINFO/07/2009 Regarding Table of Indonesian Radio Frequency Spectrum Allocation; Regulation of the Minister of Communication and Informatics: Jakarta, Indonesia, 2009; (In Indonesia Language).

7. Setiawan, D. Frequency Allocation, Policy and Spectrum Planning of Indonesia; Ministry of Communication and Informatics (Depkominfo): Jakarta, Indonesia, 2010; (in Indonesia Language).

8. Space Science Center. National Institute of Aeronautics and Space, Timau National Observatory at a Glance; Gramedia Press (PT Gramedia): Jakarta, Indonesia, 2017; (in Indonesia Language).

9. Ambrosini, R.; Beresford, R.; Boonstra, A.-J.; Ellingson, S.; Tapping, K. RFI Measurement Protocol for Candidate SKA Sites; SKA Memo No. 37; SKA Organisation: Cheshire, UK, 2003.

10. CRAF: Handbook for Radio Astronomy, 3rd ed.; European Science Foundation: Strasbourg, France, 2005.

11. National Academies Press. Handbook of Frequency Allocations and Spectrum Protection for Scientific Uses; National Academies Press: Washington, DC, USA, 2007.

12. Hidayat, T.; Munir, A.; Dermawan, B.; Jaelani, A.T.; Léon, S.; Nugroho, D.H.; Suksmono, A.B.; Mahasena, P.; Premadi, P.W.; Herdiwijaya, D.; et al. Radio frequency interference measurements in Indonesia. Exp. Astron. 2014, 37, 85-108. [CrossRef]

13. Jaroenjittichai, P.; Punyawarin, S.; Singwong, D.; Somboonpon, P.; Prasert, N.; Bandudej, K.; Kempet, P.; Leckngam, A.; Poshyachinda, S.; Soonthornthum, B.; et al. Radio frequency interference site survey for thai radio telescopes. J. Phys. Conf. Ser. 2017, 901, 12062. [CrossRef] 